\title{
Diversidade e Atividade de Voo de Himenópteros Parasitóides em Pomar de Macieira em Vacaria, RS
}

\author{
Daniela Fernanda Klesener ${ }^{\circledR}{ }^{\star}$, Régis Sivori Silva dos Santos² \& Ayres de Oliveira Menezes Jr¹.
}

1. Universidade Estadual de Londrina, e-mail: daniela.klesener@gmail.com (Autor para correspondência ${ }^{\bowtie}$ ), ayres@uel.br. 2. Embrapa Uva e Vinho, e-mail: regis.sivori@embrapa.br.

EntomoBrasilis 6(2): 108-112 (2013)

Resumo. O objetivo deste estudo foi avaliar a diversidade de himenópteros parasitóides e o período de atividade de voo em macieira. Os insetos foram amostrados com armadilha Malaise, semanalmente, por um período de 24h, sendo retirados do frasco coletor nos seguintes horários: 1, 7, 13 e 19 h. Foram coletados 1.249 himenópteros parasitóides distribuídos em 25 famílias com maior abundância de Ichneumonidae (318), Bethylidae (199), Figitidae (Eucoilinae) (183), Braconidae (90) e Scelionidae (86). Com exceção de Ichneumonidae (Ophioninae) a maioria dos parasitóides mostrou atividade de voo diurna.

Palavras-chave: Atividade circadiana; controle biológico; maçã.

\section{Diversity and Flight Activity of Parasitoids Hymenoptera in Apple Orchard in Vacaria, RS}

Abstract. The objective of this work was to evaluate the diversity of parasitoids Hymenoptera and the period of flight activity in apple trees. Insects were sampled with Malaise traps weekly for a period of 24 hours, being removed from the trap in the following times 1, 7, 13 and 19 hours. A total of 1,249 individual parasitoids from 25 families were collected. The most abundant families was Ichneumonidae (318), Bethylidae (199), Figitidae (Eucoilinae) (183), Braconidae (90) and Scelionidae (86). With the exception of Ichneumonidae (Ophioninae), parasitoids were most commonly collected during daylight.

Keywords: Apple; Biological control; Circadian activity.

] imenópteros parasitóides são utilizados em programas de controle biológico em todo o mundo para supressão de populações pragas, sendo uma alternativa ambientalmente segura aos pesticidas químicos (BECKAGE \& Drezen 2011). Todavia, o sucesso do programa estará ligado ao conhecimento bioecológico dos organismos envolvidos, bem como do aprimoramento de ações de liberação e conservação de inimigos naturais (WAAGE \& HASSELL 1982). O conhecimento das populações locais de parasitóides é passo importante para o êxito de medidas de controle biológico aplicado. Para tanto, estudos de composição faunística de parasitóides têm sido realizados com frequência em agroecossistemas brasileiros, tais como: algodão (PERioto et al. 2002a), café (РеRioto et al. 2002b), soja (РеRioto et al. 2004), sorgo, milho, feijão e trigo em rodízio (SouzA et al. 2006) e tabaco (Dorfey et al. 2011).

A informação da diversidade de parasitóides associada ao horário de maior atividade de voo dos grupos permite um melhor planejamento das ações de biorregulação e de mitigação de impactos negativo sobre a fauna benéfica. No entanto, ainda poucos são os estudos que abordam estes aspectos conjuntamente. Specht \& Corseuil (2002) em plantio de azevém verificaram diferença na abundância de alguns grupos de himenópetros parasitóides, quando amostrados pela manhã ou à noite. Em remanescente de Cerrado, MARCHIORI et al. (2007), observaram diferenças na atividade de voo de Ichneumonidae (diurno) e Encyrtidae (noturno). BRown \& SсHмiтt (2001) verificaram, em pomar de macieira na Virginia, EUA, diferenças significativas na periodicidade de ocorrência de Eulophidae, Encyrtidae e
Eucoilidae, com maior registro entre 1:20 h e 3:40 h da manhã.

Embora exista uma crescente demanda por frutas livres de resíduos de agrotóxicos, raros são os estudos de controle biológico realizados em macieira no sul do Brasil, especialmente que abordem a fauna de parasitóides existente na região. BоттоN et al. (2002) relatam três espécies de parasitóides (Braconidae e Ichneumonidae) associados a lagarta enroladeira das maçãs, com índices de parasitismo de 0,5 a 1,5\% durante a safra em Vacaria, RS. Para a mesma espécie praga, Monteiro et al. (2004), verificaram parasitismo de 22,3\% em ovos por Trichogramma pretiosum (Riley), em Fraiburgo, SC. (FonsECA 2006) estudando noctuídeos e geometrídeos pragas de macieira em Vacaria, RS, observou taxas de parasitismo de 4,55 a 9,75\% para lagartas e de 0,62 a 34,65\% para pupas, por espécies de Braconidae, Ichneumonidae, Chalcididae, Encyrtidae e Scelionidae. Independentemente dos estudos, todos os autores relataram que índices maiores de parasitismo foram encontrados em períodos de redução de aplicação de pesticidas nos pomares. Procurando agregar maior informação sobre a fauna de parasitóides existente em macieira, o presente estudo objetivou responder duas questões: 1) quais são as famílias de himenópteros parasitóides presentes em pomares de macieira? 2) o período da coleta, dia/tarde/noite, interfere na composição das famílias de himenópteros registradas?

\section{MATERIAL E MÉTODOS}

O estudo foi realizado em dois pomares de macieira cultivar Fuji, localizados na Estação Experimental de Fruticultura de Clima Temperado da Embrapa Uva e Vinho (EEFT), em Vacaria, RS 
T ( $\left.28^{\circ} 30^{\prime} \mathrm{Se}_{50}{ }^{\circ} 52^{\prime} \mathrm{W}\right)$, altitude de $970 \mathrm{~m}$, de 23 outubro de 2007 a 23 de janeiro de 2008. O pomar 1, com área de 0,11 ha, espaçamento de 4,5 x 2m e 12 anos de idade, apresenta em suas imediações pomares de macieira e área de mata nativa de, aproximadamente 4,5 ha, localizada a uma distância de cerca de $130 \mathrm{~m}$. O pomar 2, com área total de 0,33 ha, espaçamento de 4,0 x $1 \mathrm{~m}$ entre plantas de 8 anos de idade, é cercado por povoamentos de Pinus sp. de 0,34 e 0,72 ha, distantes cerca de 6 e $2 \mathrm{~m}$, respectivamente, de suas margens. Em ambos os pomares não houve aplicação de inseticidas durante o período de estudo.

Em cada pomar foram instaladas, aleatoriamente, duas armadilhas de interceptação de voo Malaise, uma no sentido perpendicular e outra paralelo às linhas de plantas (sentido lesteoeste). As armadilhas perpendiculares foram instaladas com o frasco coletor voltado para o norte, enquanto as paralelas para o leste. As amostragens foram realizadas a intervalos semanais, por um período de $24 \mathrm{~h}$ ininterruptas, retirando-se os insetos do frasco coletor, a cada 6 h, nos seguintes horários: 1, 7, 13 e 19 h (hora padrão UTC-3, desconsiderado horário de verão). Durante o período de coletas, o nascer do sol ocorreu entre 05:38 h e 05:48 h, enquanto o ocaso se deu entre 18:37 h e 19:24 h.

Os insetos coletados foram mantidos em álcool a 90\% e identificados com auxílio de chaves de identificação de GoulET \& Huber (1993). A diversidade de táxons (famílias e algumas subfamílias) foi estimada pelo Índice de Diversidade de ShanonWiener, calculado com o auxílio do programa DivEs - Diversidade de Espécies (Rodrigues 2005). Para comparar a ocorrência dos táxons capturados em relação aos pomares e horários de coleta (casual ou não), foi aplicado o teste estatístico não paramétrico de Goodman para contrastes entre proporções multinomiais entre e dentro de populações independentes, a $5 \%$ de significância. Para análise da influência do horário de coleta, utilizaram-se apenas os táxons com mais de 10 indivíduos coletados.

\section{RESULTADOS E DISCUSSÃO}

Foram coletados 1.249 himenópteros parasitóides, pertencentes a oito superfamílias e 25 famílias (Tabela 1). Destacaram-se pela abundância de indivíduos as superfamílias Vespoidea, Ichneumonoidea e Cynipoidea. Chalcidoidea apresentou menor quantidade de indivíduos, porém maior diversidade de famílias (34,5\% do total diagnosticado) (Tabela 1). Esta superfamília é rica em número de famílias e utiliza uma grande diversidade de hospedeiros (GIBSON 1993), e tem sido representativa em outros trabalhos, por exemplo, Souza et al. (2006), no qual reuniu 55\% das famílias diagnosticadas.

Tabela 1. Riqueza de táxons (superfamília, família e subfamília) de himenópteros parasitóides coletadas em armadilhas Malaise em posições perpendicular (PE) e paralela (PA) às linhas de plantas em dois pomares de macieira, cultivar Fuji. Vacaria, RS. 2007/2008.

\begin{tabular}{|c|c|c|c|c|c|c|c|}
\hline \multirow{2}{*}{ Táxons } & \multicolumn{3}{|c|}{ Pomar 1} & \multicolumn{3}{|c|}{ Pomar 2} & \multirow[b]{2}{*}{ Total } \\
\hline & PE & $\mathbf{P A}$ & $\%(n)$ & PE & PA & $\%(n)$ & \\
\hline \multicolumn{8}{|l|}{ VESPOIDEA } \\
\hline Mutillidae & 29 & o & $3(29) a^{*}$ & 7 & 8 & $5,7(15) \mathrm{b}$ & 44 \\
\hline Scoliidae & o & 4 & $0,4(4)$ & o & o & o (o) & 4 \\
\hline Tiphiidae & o & 1 & $0,1(1)$ & o & 0 & o (o) & 1 \\
\hline Bethylidae & 107 & 71 & $18(178) a$ & 2 & 19 & $8(21) b$ & 199 \\
\hline Dryinidae & 3 & 0 & $0,3(3)$ & o & o & $\mathrm{o}(0)$ & 3 \\
\hline \multicolumn{8}{|l|}{ EVANIOIDEA } \\
\hline Evaniidae & 4 & 3 & $0,7(7) a$ & 1 & 3 & $1,5(4) \mathrm{a}$ & 11 \\
\hline \multicolumn{8}{|l|}{ ICHNEUMONOIDEA } \\
\hline Ichneumonidae & 69 & 58 & 13 (127)a & 15 & 105 & $45,8(120) a$ & 247 \\
\hline Ophioninae & 9 & 3 & $1,2(12) b$ & 26 & 33 & $22,5(59) \mathrm{a}$ & 71 \\
\hline Braconidae & 18 & 24 & $4,3(42) a$ & 2 & 8 & $3,8(10) b$ & 52 \\
\hline Microgastrinae & 21 & 12 & $3,3(33) \mathrm{a}$ & o & 1 & $0,4(1) b$ & 34 \\
\hline Aphidiinae & 4 & 0 & $0,4(4)$ & o & o & o (o) & 4 \\
\hline \multicolumn{8}{|l|}{ CERAPHRONOIDEA } \\
\hline Ceraphronidae & 2 & 3 & $0,5(5)$ & o & o & o (o) & 5 \\
\hline Megaspilidae & 2 & 4 & $0,6(6)$ & o & o & o (o) & 6 \\
\hline \multicolumn{8}{|l|}{ CYNIPOIDEA } \\
\hline Figitidae & 3 & 3 & o,6 (6) & o & o & o (o) & 6 \\
\hline Eucoilinae & 93 & 79 & $17,5(172) a$ & 2 & 9 & $4,2(11) b$ & 183 \\
\hline PROCTOTRUPOIDEA & & & & & & & 0 \\
\hline Proctotrupidae & 1 & 2 & $0,3(3)$ & o & o & o (o) & 3 \\
\hline Diapriidae & 15 & 20 & $3,6(35) \mathrm{a}$ & o & 2 & $\mathrm{o}, 8(2) \mathrm{b}$ & 37 \\
\hline \multicolumn{8}{|l|}{ PLATYGASTROIDEA } \\
\hline Platygastridae & 14 & 8 & $2,2(22) a$ & o & 1 & $\mathrm{o}, 4(1) \mathrm{b}$ & 23 \\
\hline Scelionidae & 45 & 37 & $8,3(82) a$ & o & 4 & $1,5(4) b$ & 86 \\
\hline \multicolumn{8}{|l|}{ CHALCIDOIDEA } \\
\hline Chalcididae & 5 & 2 & $0,7(7)$ & o & o & o (o) & 7 \\
\hline Eurytomidae & 4 & 2 & o,6 (6) & o & o & o (o) & 6 \\
\hline Pteromalidae & 10 & 5 & $1,5(15) a$ & 0 & 2 & $\mathrm{o}, 8(2) \mathrm{b}$ & 17 \\
\hline Eucharitidae & 16 & 2 & $1,8(18) \mathrm{a}$ & o & o & $\mathrm{o}(\mathrm{o}) \mathrm{b}$ & 18 \\
\hline Eupelmidae & 1 & 1 & $0,2(2)$ & o & o & o (o) & 2 \\
\hline Encyrtidae & 19 & 17 & $3,7(36) \mathrm{a}$ & o & 3 & $1,1(3) \mathrm{b}$ & 39 \\
\hline Eulophidae & 34 & 21 & $5,6(55) \mathrm{a}$ & o & 5 & $2(5) \mathrm{b}$ & 60 \\
\hline Mymaridae & 31 & 28 & 6 (59)a & o & 3 & $1,1(3) b$ & 62 \\
\hline Aphelinidae & 6 & 9 & $1,5(15) a$ & o & 2 & $\mathrm{o}, 8(2) \mathrm{b}$ & 17 \\
\hline Trichogrammatidae & o & $\mathrm{o}$ & $\mathrm{o}(\mathrm{o})$ & $\mathrm{o}$ & 1 & $0,4(1)$ & 1 \\
\hline Total & 566 & 419 & $100(985)$ & 55 & 209 & $100(264)$ & 1249 \\
\hline
\end{tabular}

* Valores seguidos de letras distintas, na linha (comparação entre pomares para táxons mais representativos) diferem entre si pelo teste de Goodman a $5 \%$ de significância. 
O resultado para o número de famílias diagnosticadas (25) está muito próximo ao encontrado por Brown \& SchmitT (2001) em macieira nos EUA, que documentaram a presença de 396 indivíduos de himenópteros parasitóides distribuídos em 26 famílias. Além disso, registramos a ocorrência de $17(65,4 \%)$ das famílias diagnosticadas naquele estudo.

Destacaram-se pela abundância de indivíduos Ichneumonidae (318), Bethylidae (199), Figitidae (189), Braconidae (90) e Scelionidae (86) (Tabela 1). Ichneumonidae constitui-se na maior família de Hymenoptera, parasitando formas imaturas de insetos holometábolos (Guerra \& Penteado-Dias 2002). Esta família tem sido a mais representativa em vários outros estudos que utilizam armadilha Malaise para determinar a flutuação populacional de parasitóides (MARCHIORI et al. 2003; MARCHIORI et al. 2007).

Bethylidae e Figitidae foram as famílias mais abundantes no pomar 1, que, coincidentemente, tiveram o mesmo número de exemplares capturados (178) (Tabela 1). Embora os hábitos da maioria das espécies de Bethylidae sejam pouco conhecidos, geralmente atuam como ectopararasitóides idiobiontes; preferem lagartas (especialmente microlepidópteros) e larvas de coleópteros, havendo vários exemplos de seu aproveitamento no controle biológico de pragas (HAwkins \& GoRDH 1986). A família Figitidae abrange nove subfamílias que agrupam 1.411 espécies, das quais $67 \%$ pertencem a Eucoilinae (parasitóides de Diptera: Cyclorrhapha) (RonquisT 1999). A subfamília Eucoilinae já fora registrada em Vacaria, RS, por GUIMARÃEs et al. (1999) parasitando pupários da mosca-das-frutas Anastrepha sp., e apresenta espécies consideradas como importantes biocontroladores de tefritídeos no sul do Brasil (Costa et al. 2007).

Braconidae esteve entre as cinco famílias mais coletadas, representada em grande parte por espécies de Microgastrinae. Quatro espécies de braconídeos foram citadas como inimigos naturais de espécies pragas da macieira em Vacaria, RS por ВоттоN et al. (2002) e FonsECA (2006). Nesta família são encontrados importantes parasitóides de larvas de moscas-das-frutas, pulgões e, pelo menos uma espécie Macrocentrus ancylivorus (Rohwer) já fora usada no controle da mariposa oriental Grapholita molesta (Busck) (ORTH et al. 1986).

Scelionidae apresentou número de indivíduos muito próximo a Braconidae, esta família inclui parasitóides de ovos que usam espécies distribuídas em diferentes ordens como hospedeiros: Heteroptera, Neuroptera, Orthoptera, Coleoptera, Diptera e Lepidoptera (SAmin et al. 2010). BRown \& SchmitT (2001) registraram a ocorrência de Scelionidae perfazendo 11,7\% do total de parasitóides amostrados no estudo. Já FonseCA (2006) relatou parasitismo em uma postura de Peridroma saucia (Hübner) por Telenomus remus (Nixon).

Considerando-se o total de coletas, o pomar 1 apresentou maior diversidade de táxons do que o pomar 2; com menor diferença de diversidade em relação às posições das armadilha, em cada pomar (Tabela 2). Este resultado sugere, de uma forma geral, que a posição da armadilha Malaise não afetou a coleta de parasitóides, indicando voo aleatório dos grupos em pomar de macieira.

Tabela 2. Diversidade de táxons de parasitóides capturados nas diferentes posições de armadilha e entre pomares 1 e 2, através do índice de diversidade de Shanon-Wiener. Vacaria, RS, 2007/2008.

\begin{tabular}{lcc}
\hline \multirow{2}{*}{ Pomar } & \multicolumn{2}{c}{ Armadilha } \\
\cline { 2 - 3 } & Perpendicular & Paralela \\
\hline Pomar 1 & 1,1488 & 1,0772 \\
Pomar 2 & 0,6103 & 0,8593 \\
& \multicolumn{3}{c}{ Pomar } \\
\hline \multicolumn{1}{c}{ Total } & Pomar 1 & Pomar 2 \\
\hline & 0,8532 & 1,1459 \\
\hline
\end{tabular}

O fato de o pomar 1 apresentar maior diversidade de famílias pode estar relacionado à proximidade de fragmento de vegetação natural, caracterizado por grande diversidade de plantas hospedeiras, e capaz de abrigar uma gama maior de inimigos naturais, se comparada com o povoamento de Pinus sp., vizinho ao pomar 2. Algumas teorias têm sido propostas para explicar diferenças em diversidade animal, e entre elas, está o incremento na diversidade de plantas associadas aos pomares como altamente determinante (FowLER \& LAWTON 1982; SZENTKIRÁLY \& KoZÁR 1991). Esta suposição pode explicar a baixa incidência de braconídeos no pomar 2 (Tabela 1), uma vez que este grupo tem sido usado como indicador do grau de preservação de ecossistemas (RESTELLO \& Penteado-Dias 2006).

Na análise de horário de atividade de voo de 17 táxons avaliados, $15(88,2 \%)$ apresentaram atividade marcantemente diurna, com predominância de captura no período vespertino (entre 13 e 19h) seguido do período matutino (entre 7 e $13 \mathrm{~h}$ ) (Tabela 3). Uma maior coleta de parasitóides no período diurno foi relatada no estudo de Specht \& CoRseuil (2002), embora os autores tenham concentrado suas amostragens diurnas apenas no período da manhã. Em seus estudos IDRIS \& GRAFIUS (1998) verificaram que o pico de atividade de uma espécie de Ichneumonidae, Diadegma insulare (Cresson), ocorreu entre 11 e 13 h. No presente estudo, Ichneumonidae (excluindo-se a subfamília Ophioninae), Platygastridae e Scelionidade caracterizaram-se pela atividade diurna, sem diferença significativa entre o período matutino e vespertino (Tabela 3), resultados que corroboram com os apontados por (BRown \& Schmitt 2001; MARChiori et al. 2007). As famílias Diapriidae, Figitidae (Eucoilinae), Eulophidae, Mymaridae e Pteromalidae também mostraram hábito diurno, porém, preferencialmente, vespertino (Tabela 3). Embora os resultados de atividade de voo de Eulophidae e Encyrtidae (hábito diurno) sejam diferentes dos apresentados por BRown \& Sснмітт (2001) que relataram atividade significativamente noturna nos grupos, os demais resultados estão de acordo com os relatados na literatura.

Dentre as famílias com hábito diurno, a preferência pelo período matutino foi característica apenas em Eucharitidae; este grupo de parasitóides é especializado em formas imaturas de formigas e foi coletado apenas no pomar 1 (Tabelas 1 e 3).

Representantes de Bethylidae foram coletados em todos os períodos avaliados, com predominância no período vespertino (13 às $19 \mathrm{~h}$ ), seguido das primeiras horas da noite (19 à $1 \mathrm{~h}$ ), o que pode indicar a ocorrência de hábito crepuscular dentro do grupo (Tabela 3). Os Ophioninae, computados separadamente, constituíram o único grupo de atividade marcadamente noturna, com maior coleta no período inicial da noite (19 à $1 \mathrm{~h}$ ); foram também o único táxon predominante no pomar 2 (Tabelas 1 e 3). Este grupo apresenta grande diversidade de espécies, sendo que a grande maioria tem atividade noturna (GAULD 1985), quando atacam lagartas de Noctuidae (GAULD 1991).

A periodicidade de voo de himenópteros parasitóides ainda é pouco estudada, especialmente na região neotropical, onde HANSON \& GAULD (2006) relatam ter sido observada atividade noturna em algumas famílias, como Diapriidae, Ichneumonidae (especialmente Ophioninae), Braconidae (algumas subfamílias), e machos de Embolemidae e Mutillidae. Em nossas coletas, alguns braconídeos foram capturados no período noturno, porém apenas um exemplar de Diapriidae e, nenhum de Mutillidae, foi observado à noite (Tabela 3 ).

Foi constatado que apenas representantes de Ichneumonidae (excluído Ophioninae) estiveram presentes em todas as coletas. Apesar de abundantes, Ichneumonidae (Ophioninae), Figitidae (Eucoilinae) e Bethylidae foram capturados somente a partir do início de dezembro de 2007. 
Tabela 3. Número de parasitóides (n) e percentual capturados em armadilha Malaise em pomares de macieira cultivar Fuji, em função do horário de coleta. Vacaria, RS, 2007/2008.

\begin{tabular}{|c|c|c|c|c|}
\hline \multirow{2}{*}{ Famílias e subfamílias } & \multicolumn{4}{|c|}{ Faixas de horários de coleta } \\
\hline & 13 às $19 h$ & 19 à $01 h$ & 01 às $07 h$ & 07 às 13h \\
\hline Mutillidae & (26) $59 \mathrm{a}^{*}$ & (o) o b & (o) o b & (18) $41 \mathrm{a}$ \\
\hline Bethylidae & $(112) 56 \mathrm{a}$ & $(61) 31 \mathrm{~b}$ & (12) $6 \mathrm{c}$ & $(14) 7 \mathrm{c}$ \\
\hline Evaniidae & (4) $36 \mathrm{ab}$ & (o) o b & (o) o b & (7) $64 \mathrm{a}$ \\
\hline Ichneumonidae (geral) & (129) $53 \mathrm{a}$ & (11) $5 \mathrm{~b}$ & (8) $3 \mathrm{~b}$ & (95) $39 \mathrm{a}$ \\
\hline Ichneumonidae, Ophioninae & (1) $1 \mathrm{c}$ & (51) $72 \mathrm{a}$ & (18) $25 \mathrm{~b}$ & (1) $1 \mathrm{c}$ \\
\hline Braconidae (geral) & (30) $58 \mathrm{a}$ & (2) $4 \mathrm{~b}$ & (1) $2 \mathrm{~b}$ & (19) $37 \mathrm{a}$ \\
\hline Braconidae, Microgastrinae & (17) $50 \mathrm{a}$ & (3) $9 \mathrm{~b}$ & (1) $3 \mathrm{~b}$ & (13) $38 \mathrm{a}$ \\
\hline Figitidae, Eucoilinae & (112) $61 \mathrm{a}$ & (11) $6 \mathrm{c}$ & (o) o d & $(60) 33 \mathrm{~b}$ \\
\hline Diapriidae & $(32) 86 \mathrm{a}$ & (1) $3 \mathrm{~b}$ & (o) o b & (4) $11 \mathrm{~b}$ \\
\hline Platygastridae & (12) $52 \mathrm{a}$ & (o) o b & (o) o b & (11) $48 \mathrm{a}$ \\
\hline Scelionidae & $(48) 56 \mathrm{a}$ & (2) $2 \mathrm{~b}$ & (1) $1 b$ & $(35) 41 \mathrm{a}$ \\
\hline Pteromalidae & (12) $71 \mathrm{a}$ & (1) $6 \mathrm{~b}$ & (o) o b & (4) $24 \mathrm{ab}$ \\
\hline Eucharitidae & (1) $6 \mathrm{~b}$ & (o) o b & (o) o b & (17) $94 \mathrm{a}$ \\
\hline Encyrtidae & (26) $67 \mathrm{a}$ & (1) $3 \mathrm{~b}$ & (1) $3 \mathrm{~b}$ & (11) $28 \mathrm{a}$ \\
\hline Eulophidae & (47) $78 \mathrm{a}$ & (2) $3 \mathrm{~b}$ & (2) $3 \mathrm{~b}$ & (9) $15 \mathrm{~b}$ \\
\hline Mymaridae & (43) $69 a$ & (3) $5 \mathrm{c}$ & (o) o c & (16) $26 \mathrm{~b}$ \\
\hline Aphelinidae & (12) $71 \mathrm{a}$ & (o) o b & (o) o b & (5) $29 \mathrm{ab}$ \\
\hline Total & $(664) 56 a$ & (149) $12 \mathrm{c}$ & (44) $4 \mathrm{~d}$ & (339) 28 b \\
\hline
\end{tabular}

*Valores de proporção seguidos de letras distintas, na linha, diferem entre si pelo teste de Goodman a 5\% de significância.

Os parasitóides coletados no presente estudo foram adultos em atividade de voo nos pomares, o que não nos permitiu obter taxas de parasitismo. Contudo, a abundância de famílias existentes indica que pomares de macieira são habitat para muitos parasitóides, sugerindo uma ação biorreguladora dos grupos. Além disso, diagnosticamos que a atividade de voo varia em função dos táxons, porém com predominância no período vespertino. Este resultado, associado ao período de realizações de intervenções com pesticidas nos pomares (durante o dia) limita a expressão desta diversidade de inimigos naturais em termos de taxas de parasitismo, conforme já observado nos estudos de BotTon et al. (2002); Monteiro et al. (2004) e FonseCA (2006) na região produtora de maçã.

Apesar disto, ações de controle biológico com parasitóides podem ser uma alternativa para a exploração econômica da macieira, entretanto, informações sobre as espécies ocorrentes dentro das famílias diagnosticadas, formas de multiplicação, conservação e seletividade a pesticidas ainda devam ser investigados.

Podemos concluir que pomares de macieira sem aplicação de inseticidas são habitat para um complexo de famílias de himenópteros parasitoides e também que o horário de atividade de voo no pomar varia em função da família do parasitóide, com certa predominância pelo período vespertino do dia.

\section{AGRADECIMENTOS}

À professora Dra. Inês Cristina de Batista Fonseca (Departamento de Agronomia, da Universidade Estadual de Londrina), e ao colega biólogo MSc. Fábio Henrique Dangui pela ajuda na realização das análises estatísticas.

\section{REFERÊNCIAS}

Beckage, N.E. \& J.M. Drezen, 2011. Parasitoid Viruses: Symbionts and Pathogens. London, Academic Press, 312p.

Botton, M., O. Nakano \& A. Kovaleski, 2002. Parasitóides associados à lagarta-enroladeira Bonagota cranaodes (Meyrick, 1937) (Lepidoptera: Tortricidae) na cultura da macieira. Ciência Rural, 32: 341-343.

Brown, M.W. \& J.J. Schmitt, 2001. Seasonal and diurnal dynamic of beneficial insect populations in apple orchards under different management intensity. Environmental Entomology, 30: 415-424.

Costa, R.I.F., C.G. Silva, C.H. Marchiori, B.B. Amaral, M.M. Poletti \& L.C. Torres, 2007. Parasitismo em Anastrepha sp. (Diptera: Tephritidae) por Aganaspis pelleranoi (Brèthes, 1924) e Dicerataspis sp. (Hymenoptera: Figitidae: Eucoilinae). Ciência e Agrotecnologia, 31: 720-723.

Dorfey, C., K. Schoeninger \& A. Köhler, 2011. Levantamento das famílias de himenópteros parasitóides associados ao cultivo de tabaco (Nicotiana tabacum L.) em Santa Cruz do Sul e Lagoão, Rio Grande do Sul, Brasil. Arquivos do Instituto Biológico, 78: 449-451.

Fonseca, F.L., 2006. Ocorrência, monitoramento, caracterização de danos e parasitismo de Noctuidae e Geometridae em pomares comerciais de Macieira em Vacaria, Rio Grande do Sul, Brasil. Tese (Doutorado em Ciências: Entomologia) Universidade Federal do Paraná, Curitiba, 97p.

Fowler, S.V. \& J.H. Lawton, 1982. The effect of host-plant distribution and local abundance on the species richness of agromyzid flies attacking British umbellifers. Ecological Entomology, 7: 257-265.

Gauld, I., 1991. The Ichneumonidae of Costa Rica. Memoirs of the American Entomological Institute, 47:1-589.

Gauld, I., 1985. The phylogeny, classification and evolution of parasitic wasps of the subfamily Ophioninae(Ichneumonidae). Bulletin of the British Museum (Natural History), 51: 61-185.

Gibson, G.A.P., 1993. Superfamilies Mymarommatoidea and Chalcidoidea, p.570-655. In: Goulet, H.; Huber J. T. (Eds). Hymenoptera of the world: an identification guide to families. Ontario: Agriculture Canada Publication, 668p.

Goulet, H. \& J.T. Huber, 1993. Hymenoptera of the world: an identification guide to families. Ontario: Agriculture Canada Publication, 668p.

Guerra, T.M. \& A.M. Penteado-Dias, 2002. Abundância de Ichneumonidae (Hymenoptera) em área de mata em São Carlos, Estado de São Paulo, Brasil. Acta Scientiarum, 24: 363-368.

Guimarães, J.A., R.A. Zucchi, N.B. Diaz, M.F. Souza Filho \& M.A.F. Uchôa, 1999. Espécies de Eucoilinae (Hymenoptera: Cynipoidea: Figitidae) parasitóides de larvas frugívoras (Diptera: Tephritidae e Lonchaeidae) no Brasil. Anais da Sociedade Entomológica do Brasil, 28: 263-273.

Hanson, P.E. \& I. Gauld, 2006. La biologia de los himenópteros. Cap. 2. In: Hymenoptera de la región Neotropical. Memoirs of the American Entomological Institute, 77: 1-994.

Hawkins, B.A. \& Y.G. Gordh, 1986. Bibliography of the world literature of the Bethylidae (Hymenoptera: Bethyloidea). Insecta Mundi, 1: 261-283.

Idris, A.B. \& E. Grafius, 1998. Diurnal flight activity of Diadegma insulare (Hymenoptera: Ichneumonidae), a parasitoid of the 
Diamondback Moth (Lepidoptera: Plutellidae), in the field. Environmental Entomology, 27: 406-414.

Marchiori, C.H., L.A. Pereira, V.R. Borges, L.C.S. Ribeiro, O.M. Silva Filho, N.B. Diaz \& F. Gallardo, 2003. Parasitóides da subfamília Eucoilinae (Hymenoptera: Figitidae) coletados em armadilhas de bacias amarelas e armadilhas Malaise em Araporã, Minas Gerais e Itumbiara, Goiás. Arquivos do Instituto Biológico, 70: 207-209.

Marchiori. C.H., M.A Lussari, D.C. Rosa \& A.M. Penteado-Dias, 2007. Parasitoid Hymenoptera collected during the diurnal and nocturnal periods in Itumbiara, Goiás. Brazilian Journal of Biology, 67: 581-582.

Monteiro, L.B., A. Souza, E.L. Belli, R.B.Q. Silva \& R.A. Zucchi, 2004. Ocorrência de Trichogramma pretiosum Riley (Hymenoptera: Trichogrammatidae) em ovos de Bonagota cranaodes (Meyrick) (Lepidoptera: Tortricidae) em macieira. Revista Brasileira de Fruticultura, 26: 171-172.

Orth, A. I., L.G. Ribeiro \& W.R. Filho, 1986. Manejo de pragas, p. 341-379. In: Buss, A. (Ed.). Manual da cultura da Macieira. Florianópolis: Empresa Catarinense de Pesquisa Agropecuária, 562p.

Perioto, N.W., R.I.R. Lara, J.C.C. Santos \& A. Selegatto, $2002 a$. Himenópteros parasitóides (Insecta, Hymenoptera) coletados na cultura de algodão (Gossypium hirsutun L.) (Malvaceae), no município de Ribeirão Preto, SP, Brasil. Revista Brasileira de Entomologia, 46: 165-168.

Perioto, N.W., R.I.R. Lara, J.C.C. Santos \& T.C. Silva, 2002b. Himenópteros parasitóides (Insecta, Hymenoptera) coletados na cultura de soja (Glycine max (L.) Merril) (Fabaceae), no município de Nuporanga, SP, Brasil. Revista Brasileira de Entomologia, 46: 185-187.

Perioto, N. W., R.I.R. Lara, A. Selegatto \& E.S. Luciano, 2004. Himenópteros parasitóides (Insecta, Hymenoptera) coletados na cultura de café Coffea arabica L. (Rubiaceae) em Ribeirão
Preto, SP, Brasil. Arquivos do Instituto Biológico, 71: 41-44.

Restello, R.M. \& A.M. Penteado-Dias, 2006. Diversidade dos Braconidae (Hymenoptera) da Unidade de Conservação Teixeira Soares, Marcelino Ramos, RS, com ênfase nos Microgastrinae. Revista Brasileira de Entomologia, 50: 8084.

Rodrigues, W.C., 2005. DivEs - Diversidade de espécies. Versão 2.o. Software e Guia do Usuário. Disponível em $<$ http://www.ebras.bio.br>.

Ronquist, F., 1999. Phylogeny, classification and evolution of the Cynipoidea. Zoologica Scripta, 28: 139-164.

Samin, K., M. Shojai, H. Ghahari \& E. Kocak, 2010. A contribution to the scelionid wasps (Hymenoptera: Platygastroidea: Scelionidae), egg parasitoid of Pentatomidae (Heteroptera) in Tehran province, Iran. Efflatounia, 10: 7-14.

Souza, L., S.M.P. Braga \& M.J.O. Campos, 2006. Himenópteros parasitóides (Insecta: Hymenoptera) em área agrícola de Rio Claro, SP, Brasil. Arquivos do Instituto Biológico, 73: 465469.

Specht, A. \& E. Corseuil, 2002. Avaliação populacional de lagartas e inimigos naturais em azevém, com rede de varredura. Pesquisa Agropecuária Brasileira, 37: 1-6.

Szentkirály, F. \& F. Kozár, 1991. How many species are there in apple insect communities?: testing the resource diversity and intermediate disturbance hypotheses. Ecological Entomology, 16: 491-503.

Waage, J.K. \& M.P. Hassell, 1982. Parasitoids as biological control agents: a fundamental approach. Parasitology, 84: 241-268.

\section{Recebido em: 09/o7/2012}

Aceito em: 28/o3/2013

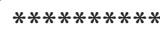

\section{Como citar este artigo:}

Klesener, D.F., R.S.S. Santos \& A.O. Menezes Jr., 2013. Diversidade e Atividade de Voo de Himenópteros Parasitóides em Pomar de Macieira em Vacaria, RS. EntomoBrasilis, 6(2): 108-112.

Acessível em: http://www.periodico.ebras.bio.br/ojs/index.php/ebras/article/view/258. doi:10.12741/ebrasilis.v6i2.258

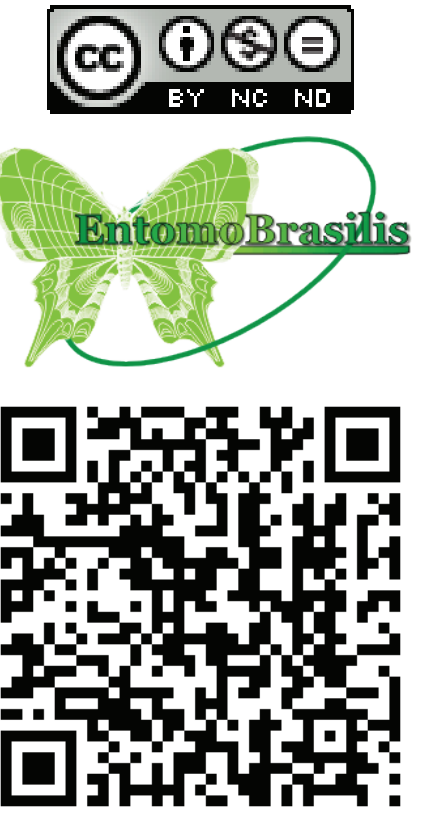

